\title{
Searching for Common Ground: Existing Literature on Automotive Agile Software Product Lines
}

\author{
Philipp Hohl, Javad Ghofrani, Jürgen Münch, Michael Stupperich, Kurt Schneider ${ }^{5}$
}

This summary refers to the paper Searching for Common Ground: Existing Literature on Automotive Agile Software Product Lines [Ho17]. This paper was published in the Proceedings of the International Conference on Software and System Process (ICSSP 2017).

Keywords: software product line; agile software development; automotive domain

The need for creating digitally enhanced products, services, and experiences as well as the emergence of new or modified business models has a significant impact on the automotive domain. Innovative solutions and new topics such as Smart Mobility or Connectivity require current automotive development processes to undergo major changes. They need to be redesigned in a way that it is possible to learn and adapt continuously at a fast pace. Agile methods are promising approaches to address these new challenges. However, agile methods are not tailored to the specific characteristics of the automotive domain such as software product line (SPLs) development. Although, there have been efforts to apply agile methods in the automotive domain, widespread adoptions have not yet taken place.

We conducted a comprehensive literature study [Ho17] that gives an overview of agile methods for embedded software development in the automotive domain, especially with respect to SPLs. We limited the scope to automotive embedded software development. Typical characteristics of the automotive domain that need to be considered are the deep integration between hardware and software, a strong focus on development processes, a close supplier involvement, and the importance of safety-critical functionality. Furthermore, specific testing conditions like tests in real cars must be considered. The general research question that guides the study is: What is the state-of-the-art to combine agile software development and software product lines in the automotive domain, according to published literature? This question is divided into three research sub-questions to provide different views on the topic (see Fig. 1).

\footnotetext{
${ }^{1}$ Daimler AG, Wilhelm-Runge-Straße 11, 89013 Ulm, Germany, Philipp.Hohl@ daimler.com

${ }^{2}$ Leibniz Universität Hannover, Welfengarten 1, 30167 Hannover, Germany, javad.ghofrani@inf.uni-hannover.de

${ }^{3}$ University of Reutlingen, Danziger Str. 6, 71034 Böblingen, Germany, juergen.muench@ reutlingen-university.de

${ }^{4}$ Daimler AG, Wilhelm-Runge-Straße 11, 89013 Ulm, Germany, Michael.Stupperich@ daimler.com

${ }^{5}$ Leibniz Universität Hannover, Welfengarten 1, 30167 Hannover, Germany, kurt.schneider@inf.uni-hannover.de
} 


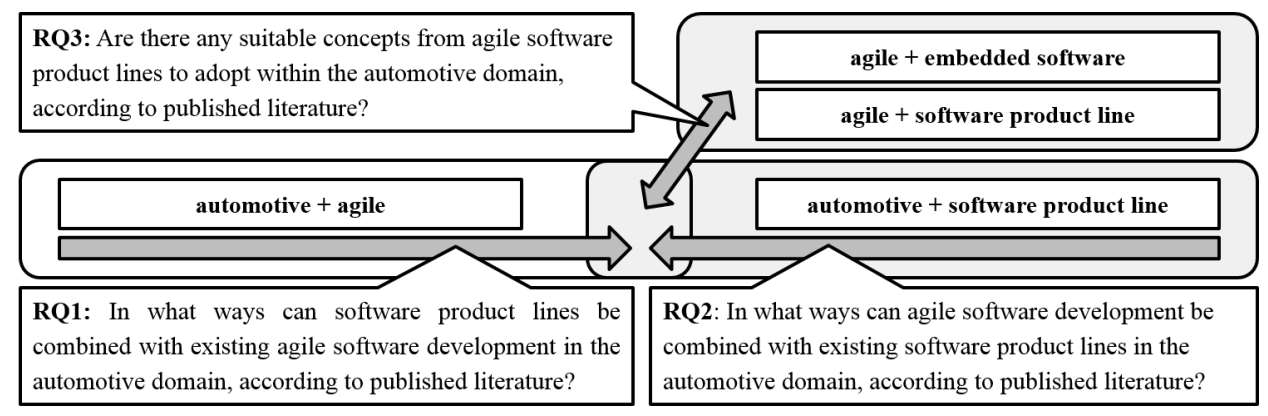

Fig. 1: What is the state-of-the-art to combine agile software development and software product lines in the automotive domain, according to published literature?

The literature review revealed that there is no specific approach tailored to the automotive domain handling the combination of agile software development (ASD) and SPLs. A possible explanation is that combining agile development with existing product lines is challenging in the automotive domain (consider, for instance, the need for synchronization with mechanical and electrical engineering). Searching for existing literature about the introduction of agile methods in the context of automotive product line development was also not fruitful. The study identified areas in the automotive domain where it seems to be comparably easy to introduce agile development without product line development (e.g., entertainment software for the car). Searching for literature that deals with introducing product line engineering to agile development in the automotive domain also did not reveal significant results. However, looking at related domains provided many insights into how the combination of agile methods and product line development could look alike in the automotive domain.

The study identified challenges and possible solutions to combine ASD with SPL. Examples for such solutions are approaches for architecture evolution, lightweight variability management, new communication structures and practices, lean and incremental safetyrelated procedures, and advanced synchronization mechanisms for hardware and software development cycles. The insights from the study might help as starting points to modify automotive software development processes so that they can benefit from both, agility and systematic reuse through product lines.

\section{References}

[Ho17] Hohl, P.; Ghofrani, J.; Münch, J.; Stupperich, M.; Schneider, K.: Searching for common ground: Existing literature on automotive agile software product lines. In (Bendraou, R.; Raffo, D.; LiGuo, H.; Maggi, F. M., eds.): Proceedings of the 2017 International Conference on Software and System Process - ICSSP 2017. ACM Press, New York, New York, USA, pp. 70-79, 2017, Is B N: 9781450352703. 